\title{
Pharmacological mechanism of roflumilast in the treatment of asthma-COPD overlap
}

This article was published in the following Dove Press journal:

Drug Design, Development and Therapy

\author{
Xiaoli Zhang ${ }^{1, *}$ \\ Yuqing Chen ${ }^{1, *}$ \\ Liyu Fan ${ }^{1, *}$ \\ Jiaqi Ye' \\ Junsheng Fan² \\ Xinjie $\mathrm{Xu}{ }^{\prime}$ \\ Danming You ${ }^{3}$ \\ Sihan $\mathrm{Liu}^{3}$ \\ Xin Chen' \\ Peng Luo'
}

'Department of Respiratory Medicine, Zhujiang Hospital of Southern Medical University, Guangzhou, China; ${ }^{2}$ Department of Respiratory Medicine, Shanghai Tenth People's Hospital of Tongji University, Shanghai, China; ${ }^{3}$ Department of Respiratory Medicine, Nangfang Hospital of Southern

Medical University, Guangzhou, China

*These authors contributed equally to this work
Correspondence: Peng Luo

Zhujiang Hospital of Southern Medical University, 253 Industry Road,

Guangzhou, Guangdong 510280, China

Tel +86 I 3246269394

Fax +862061643888

Email luopeng@smu.edu.cn

Xin Chen

Zhujiang Hospital of Southern Medical

University, 253 Industry Road,

Guangzhou, Guangdong 510280, China

Tel +862062782296

$\mathrm{Fax}+862061643010$

Email chen_xin1020@163.com

\begin{abstract}
Asthma-COPD overlap (ACO) is a type of incomplete obstructive airway disease that has a high incidence and mortality. Nevertheless, there is currently no clear definition of ACO and no effective intervention. The newly discovered phosphodiesterase-4 inhibitor, roflumilast, has shown initial efficacy for treating asthma, COPD, and ACO. The mechanism of roflumilast, however, remains unclear, and there has been no interpretation through systematic review to date. The determination of a definite mechanism of roflumilast will guide the clinician's decisions regarding medication use, standardized diagnosis, and treatment guidelines. For this reason, we have systematically reviewed the therapeutic mechanism of roflumilast for ACO and provided reference for the clinical application of roflumilast in ACO.
\end{abstract}

Keywords: asthma-COPD overlap, roflumilast, phosphodiesterase-4 inhibitors, mechanism

\section{Introduction}

COPD is characterized by persistent airflow limitation, which is always progressive. ${ }^{1}$ Asthma is a heterogeneous disease characterized by chronic airway inflammation accompanied by variable respiratory flow limitation, ${ }^{2}$ whereas asthma-COPD overlap (ACO) is characterized by persistent airflow limitation associated with features related to asthma and COPD. ACO is a type of persistent airflow limitation that has several features mainly associated with asthma and COPD. It encompasses several clinical phenotypes and may have a variety of potential pathogenesis. ${ }^{2}$ The incidence of ACO in obstructive airway diseases is $\sim 15 \%-25 \%,{ }^{3,4}$ and ACO shows regional differences. In Italy, South Korea, Latin America, and the USA, the incidence of ACO varies from $1.6 \%$ to $4.5 \%$. In Asia, ACO patients are mostly elderly and male. ${ }^{5}$ ACO can be broadly defined ${ }^{6}$ as COPD with reversible characteristics or asthma with partial reversibility of airflow limitation. ${ }^{7}$ ACO clinically manifests as obstructive ventilatory dysfunction, which shows similarities to asthma and COPD, but also has its own characteristics. In terms of airway remodeling, it is similar to COPD; ${ }^{8}$ in terms of airway inflammation, ACO has the features of both eosinophil-based asthma inflammation and neutrophil-based COPD inflammation. ${ }^{9}$ Compared with COPD and asthma, there is a high incidence of ACO in people over the age of 40 years, who may have had corresponding symptoms in childhood or early adulthood. ${ }^{10}$ In these individuals, acute exacerbation occurs more frequently and the exacerbation frequency is 2-2.5 times that of COPD. ${ }^{11}$ Additionally, compared with patients with asthma, patients with ACO show higher levels of eosinophils, which can reach as high as $300 \mu \mathrm{L},{ }^{12}$ higher serum total immunoglobulin E levels, and higher nitric oxide levels in the expiratory air, ${ }^{13}$ while the periostin level remains low. ${ }^{14}$ Comparing the characteristics between ACO, asthma, and COPD is critical for determining a clinical classification and diagnosis. 
Methods for improving pulmonary ventilation and inflammatory response in patients with ACO include vaccination, antiinfection strategies, encouraging patients to quit smoking, inhaled corticosteroid treatment, ${ }^{15,16}$ long-acting beta agonists, 5-lipoxygenase inhibitor, long-term home oxygen therapy, etc. ${ }^{4,17}$ Moreover, Louie et $\mathrm{al}^{10}$ found that Omaza monoclonal antibody has a certain effect on severe asthma with ACO. ${ }^{7}$ However, the current treatment methods are limited and their efficacy is unsatisfactory. Medications for ACO continue to be studied. Although there are other effective targets in ACO treatment, such as airway remodeling, hyperresponsiveness, and fibrosis, anti-inflammatory therapy is always the core treatment and is currently recognized as the best therapy. ${ }^{13}$

Several selective phosphodiesterase (PDE) inhibitors, such as PDE-3, PDE-4, PDE-5, and PDE-7, have been successfully used in the laboratory and in clinical studies. PDE-5 inhibitors are promising agents for treating an allergic inflammation, but their effect is significantly lower than that of PDE-4 inhibitors. PDE-5 inhibitors are mainly used to treat erectile dysfunction, pulmonary hypertension, and other cardiovascular diseases. ${ }^{18}$ Presently, roflumilast and cilomilast, the PED-4 inhibitors, are already in Phase III clinical trials; of the two, roflumilast and its main metabolites, such as roflumilast- $N$-oxide, show higher selectivity and better tolerance. Furthermore, its curative effects on COPD and allergic asthma have also been confirmed in experimental and clinical conditions, ${ }^{19}$ thus, roflumilast could have curative effects in the treatment of ACO. The specific effects of ACO, asthma, and COPD are shown in Table 1. The main mechanism of PDE-4 inhibitors is that they inhibit the production of cytokines, cell proliferation, and chemotaxis; the release of inflammatory mediators; and the activity of NADPH oxidase by increasing the cyclic adenosine monophosphate (cAMP) endocellular concentration. ${ }^{20}$ In recent years, various studies have shown that cAMP is an important regulating substance of cellular function and plays a key role in the realization of cellular metabolism and various physiological effects. Inagaki et $\mathrm{al}^{21}$ indicated that a high level of cAMP can inhibit the release of histamine, lysosomal enzyme, oxygen free radical, bradykinin, eosinophil chemotactic factors, and so on to reduce bronchospasm. Moreover, cAMP could effectively inhibit the release of other inflammatory mediators and improve the permeability of blood vessels. Thus, the PDE-4 inhibitor would have a good effect on ACO without doubt. The treatment effects are primarily through the selectivity of PDE-4B and PDE-4D subtype receptors. PDE-4B is the main subtype receptor mediating anti-inflammatory effects, ${ }^{22}$ while PDE-4D induces side effects, such as nausea and vomiting, by stimulating neurons in the vomiting center.

Roflumilast is a long-acting, oral PDE-4 inhibitor. It has a significant effect on inflammation produced by eosinophils and neutrophils, airway remodeling, and bronchoconstriction ${ }^{23,24}$ and has achieved good results for treating patients with COPD and asthma. Although it has not been widely used clinically at present, its clinical treatment effect cannot be ignored. Studies have shown that taking $500 \mathrm{mg}$ of roflumilast in the morning or at night has a significant clinical effect on mild to severe asthma or COPD. ${ }^{25}$ Roflumilast is the only approved PDE-4 inhibitor that can be used to treat patients with COPD along with chronic cough and sputum, and it is the best agent for treating acute exacerbation of COPD with bronchitis. ${ }^{26}$ Data show that roflumilast can reduce the risk of acute exacerbation from $15.5 \%$ to $11.9 \%$ in patients with COPD, ${ }^{27,28}$ thereby reducing the likelihood of acute exacerbation, ${ }^{29}$ postponing the deterioration of COPD, and properly increasing the forced expiratory volume in 1 second $\left(\mathrm{FEV}_{1}\right)$. Roflumilast can also reduce deterioration of moderate and severe COPD and improve pulmonary function. ${ }^{30,31}$ Previous research has proven that roflumilast can lead to an average increase of $53.52 \mathrm{~mL}$ in $\mathrm{FEV}_{1} \cdot{ }^{32}$ Several clinical trials have demonstrated that roflumilast could mildly, but significantly, increase $\mathrm{FEV}_{1}$ levels in patients with COPD. ${ }^{33-35}$ Additionally, the St George's score decreased significantly in patients treated with roflumilast. Additionally, roflumilast can act on fibroblasts to reduce the airway fibrosis level, and inhibit eosinophils from releasing oxygen

Table I Role of roflumilast in asthma alone, COPD alone, and ACO

\begin{tabular}{|c|c|c|}
\hline Asthma & COPD & $\mathrm{ACO}$ \\
\hline I. Reduces airway inflammation & I. Reduces airway inflammation & I. Reduces airway inflammation \\
\hline 2. Reduces bronchoconstriction & 2. Improves airway remodeling & 2. Enhances mucociliary clearance \\
\hline 3. Enhances mucociliary clearance & 3. Improves pulmonary ventilation function & 3. Relieves airway hyperresponsiveness \\
\hline 4. Improves airway remodeling & 4. Reduces oxygen free radical release & 4. Improves airway remodeling \\
\hline \multirow[t]{4}{*}{ 5. Relieves airway hyperresponsiveness } & 5. Inhibits pulmonary fibrosis & 5. Improves pulmonary ventilation function \\
\hline & & 6. Reduces bronchoconstriction \\
\hline & & 7. Reduces oxygen free radical release \\
\hline & & 8. Inhibits pulmonary fibrosis \\
\hline
\end{tabular}

Abbreviation: ACO, asthma-COPD overlap. 
free radicals, ${ }^{36}$ reduce mucus secretion, etc. Pan et al found that roflumilast is likely to improve the risk of dyspnea in patients with COPD. ${ }^{37}$ The current side effects of roflumilast include diarrhea (6.8\%), weight loss $(5.2 \%)$, nausea $(3 \%),{ }^{23}$ nasopharyngitis, influenza, respiratory tract infection, etc, ${ }^{17}$ but the risk is low and the causes of these side effects remain unknown. ${ }^{25}$ In patients with asthma, studies have shown that oral roflumilast use can significantly reduce airway hyperresponsiveness (AHR; 1,000 $\mu \mathrm{g} /$ day), ${ }^{38}$ improve bronchial inflammatory cell infiltration, reduce the levels of interleukin (IL)-6 and tumor necrosis factor (TNF)- $\alpha,{ }^{39,40}$ thus further alleviating the patient's airway inflammation, airway remodeling, and bronchoconstriction by acting on eosinophils. Moreover, studies have demonstrated that roflumilast can expand the bronchial apoptosis of inflammatory cells and thus improve patients' quality of life. ${ }^{5}$ A random, double-blinded, placebo-controlled trial indicated that patients receiving any dose of roflumilast exhibited a significant decrease in their response to both early and terminal asthma induced by allergens. ${ }^{41}$ Another clinical trial that included 690 asthma patients showed that roflumilast could significantly increase the $\mathrm{FEV}_{1}$ and increase the morning peak expiratory flow in a dose-dependent manner. Additional clinical trials are needed to further support these facts. ${ }^{42}$ Currently, other studies have documented that roflumilast helps improve the efficacy of other agents; for instance, roflumilast and its $\mathrm{N}$-oxide can enhance the activity of glucocorticoid receptors and the transcription of the glucocorticoid-dependent gene. Additionally, compared with the stand-alone use of roflumilast, the combination of roflumilast and fluticasone can significantly reduce AHR in asthmatic patients. ${ }^{25}$

With its complex etiology, ACO has a relatively high morbidity and mortality. ${ }^{31}$ Compared with either COPD or asthma alone, the death risk of ACO is higher. ${ }^{43}$ Roflumilast is considered a new and effective method for treating ACO both in vivo and in vitro. ${ }^{44}$ Existing studies show that the mechanism of roflumilast is complex. It can either significantly reduce the number of eosinophils (by $\sim 42 \%$ ) and neutrophils (by $\sim 31 \%$ ) in the sputum or act on some cytokines to alleviate airway inflammation, airway remodeling, and other symptoms. ${ }^{28}$ Also, roflumilast leads to increase of cAMP and restrains the inflammatory cells such as neutrophils, eosinophils, macrophages, and $\mathrm{T}$ cells to release cytokines and chemokines. Moreover, when roflumilast interacts with the macrophages directly, it would cause decrease in the release of IL-1 $\beta$, IL-10, and nitric oxide. Although the clinical use of roflumilast in patients with ACO has shown some results, ${ }^{32}$ its specific mechanism remains unclear; thus, a more systematic review is needed for further clarification of the mechanism of roflumilast. A clear mechanism of action will undoubtedly contribute to effective clinical decision making regarding medications, standardized diagnosis, and treatment guidelines. Based on the existing research, we aim to classify and systematically summarize the mechanism of action of roflumilast.

\section{Treatment mechanisms of roflumilast Airway inflammation}

In 2014, on examining the levels of C-reactive protein and IL-6 in patients with ACO and comparing the results with the corresponding levels in patients with COPD and asthma, researchers found that the levels in patients with ACO were higher than those in patients with asthma, which were similar to those in patients with COPD, suggesting that the systemic inflammation in ACO was very similar to that in COPD. ${ }^{45}$ Asthmatic inflammation is an eosinophil-based chronic inflammatory response that is mediated by $\mathrm{CD}^{+} \mathrm{T}$ cells. At the same time, this inflammation manifests as an extremely reversible airflow limitation caused by IL-4, IL-5, IL-13, and other inflammatory mediators. The inflammation in COPD manifests as airway inflammation and pulmonary structural damage caused by $\mathrm{CD} 8^{+} \mathrm{T}$ cell-mediated, neutrophil-based chronic inflammation, accompanied by IL-6, IL-8, IL-16, TNF- $\alpha$, etc. It is known that ACO inflammation has the characteristics of both eosinophil-based asthma inflammation and neutrophil-based COPD inflammation. ${ }^{38}$ The new PDE-4 inhibitor roflumilast can not only directly or indirectly act on inflammatory cells, such as eosinophils and neutrophils, but also improve the airway inflammation of ACO by promoting salbutamol-induced clearance of inflammatory mediators in the airway or other approaches and affect the levels of certain inflammatory mediators. ${ }^{46}$ The effects of roflumilast on major airway inflammatory indicators of ACO are summarized below to show roflumilast's potential for treating ACO airway inflammation.

\section{Interleukin-6}

The study of Barnes et $\mathrm{al}^{55}$ showed that roflumilast could reduce the level of IL-6 in patients with ACO and eventually alleviate ACO inflammation. This effect may be related to the following factors. A decrease in IL-6 level leads to a decrease in the expression of RoRrt in the nasal mucosa, thereby inhibiting the accumulation, activation, or differentiation of T helper 17 (Th17) lymphocytes and ultimately reducing IL-17A and IL-17F levels. Thus, the recruitment of 
neutrophils is inhibited, resulting in the reduction of airway inflammation. ${ }^{47}$ Additionally, a decrease in IL-6 level would make it more difficult for the T cells to differentiate into Th17 cells, thereby reducing the IL-17 level, which in turn would decrease the content of IL-6, IL-8, granulocyte-macrophage colony stimulating factor (GM-CSF), and matrix metallopeptidase 2 and ultimately relieve the ACO symptoms.

\section{Interleukin-8}

Grootendorst et al used IL-8 as a marker of inflammatory cell activation to study the efficacy of roflumilast against COPD. The results showed that the level of $1 \mathrm{~L}-8$ in patients with COPD who have taken roflumilast was decreased, and that the cell activity in the respiratory tract was reduced. ${ }^{48}$ Previous studies have proven that IL-8 can gather neutrophils by acting on them and, thus, induce chemotaxis, which can attract more inflammatory cells, finally resulting in inflammation. Therefore, the reduction of IL-8 level will undoubtedly alleviate the inflammation of ACO and support the antiinflammatory effect of roflumilast. Additionally, Gauvreau et $\mathrm{al}^{9}$ conducted an experiment comparing the results of roflumilast and placebo in 14-day asthma treatment. ${ }^{9}$ The results showed that increases in the total quantity of allergen-induced eosinophils, neutrophils, metachromatic cells, and leukocytes in the sputum of the patients treated with roflumilast were inhibited. The main mechanism of this inhibition may be that roflumilast inhibits the expression of $\mathrm{P}$-selectin, $\mathrm{P}$ - and E-selectin in the vascular endothelial cells, and CD11b, L-selectin, lymphocyte function-associated antigen-1 in the neutrophils and their binding to the corresponding ICAM receptors, further inhibiting the interactions of cell to cell, blocking the inflow of inflammatory cells. At the same time, the release of various inflammatory mediators, including IL-8, is blocked, the level of IL-8 is decreased, neutrophil aggregation is inhibited, and chemotaxis to other inflammatory cells is reduced. ${ }^{37}$

\section{Interleukin- I7}

It has been recently discovered that IL-17 initiates several inflammatory factors. Several studies have shown that roflumilast can reduce the IL-17 level, thereby alleviating ACO airway inflammation. ${ }^{16}$ IL-8 plays an important role in the pathogenesis of COPD. It can not only stimulate inflammatory cytokines such as IL-6, IL-8, GM-CSF, and matrix metallopeptidase 2 and cause inflammation in COPD but also induce airway secretion of mucus, promote airway remodeling, and eventually lead to pathological changes in its target organs. ${ }^{49}$ There are several studies on IL-17. Ogura et al believed that IL- 6 can promote the initial $\mathrm{T}$ cell in patients with COPD differentiation into Th17 cells, resulting in a high level of IL-17, and that IL-17 can stimulate airway epithelial cells and fibroblasts of the lung to produce more IL-6. ${ }^{50}$ Both inflammatory factors promote each other, leading to inflammation cascade amplification, and ultimately cause irreversible damage. Jianli et $\mathrm{al}^{56}$ explored the clinical value of IL-17, which is the representative cytokine of peripheral blood $\mathrm{CD}^{+} \mathrm{T}$ cell subset Th17, in the differential diagnosis of chronic airway inflammatory diseases such as asthma and ACO. They found that the Th17 cytokine IL-17 was significantly higher in the asthma and ACO group than that in the COPD group $(P<0.01)$. It is speculated that IL-17 may be an indicator of airway inflammation. A series of studies have shown that roflumilast can reduce the level of chronic obstructive diseases such as asthma and COPD. Additionally, some studies have proven that the serum concentrations of IL-23 and IL-17 in patients with severe bronchial asthma before treatment were higher than those in healthy subjects. Roflumilast demonstrated a better effect in reducing the level of IL-17, thus reducing IL-17's promotion of airway inflammation in ACO. Therefore, roflumilast can alleviate the symptoms of airway inflammation in patients with ACO by reducing the level of IL-17. Roflumilast can reduce the level of IL-17 by many mechanisms, such as inhibiting the activation of NF-kB through increasing cAMP concentration, thereby inhibiting the accumulation or activation of Th17 cells, thus resulting in a decrease in the levels of IL-17A and IL-17F.

\section{TNF- $\alpha$}

TNF- $\alpha$ is mainly involved in causing airway inflammation in ACO by aggregating neutrophils in the lungs. Current studies have shown that roflumilast can improve airway inflammation in ACO by reducing the level of TNF- $\alpha$ in a variety of ways. The main avenues by which roflumilast can affect TNF- $\alpha$ are as follows:

1. Roflumilast inhibits the proliferation of $\mathrm{CD}^{+} \mathrm{T}$ cells and reduces the number of $\mathrm{Th} 1, \mathrm{Th} 2$ cells, thereby reducing the production of IL-4, IL-5, IL-13, IPN-r, lymphotoxin and the levels of eosinophils induced by allergen in the sputum and inhibiting the activation of eosinophils. This inhibition of activation can directly reduce inflammation in ACO and affect the activation of P38 MAPK by inhibiting the release of reactive oxygen and the production of oxygen free radicals in eosinophils, thereby reducing the chemotaxis of neutrophils and decreasing the level of TNF- $\alpha$ and calcium. 
2. Increased concentration of cAMP can inhibit the DNA binding activity of NF- $\kappa \mathrm{B}$ by preventing the phosphorylation and degradation of $\mathrm{NF}-\kappa \mathrm{B}$ inhibitory factors. The DNA binding activity of NF- $\kappa \mathrm{B}$ decreases, thereby inhibiting the gene transcription of TNF- $\alpha$ and IL- $1 \beta$ and decreasing the production and release of TNF- $\alpha$ and IL- $1 \beta$.

3. The increase in cAMP concentration can inhibit the production of GM-CSF and reduce the expression of CCL2, CCL3, CCL4, CXCL10, and IL-1 $\beta$ in the lung macrophages, which are induced by lipopolysaccharides, and TNF- $\alpha$, eventually leading to a reduced level of TNF- $\alpha \cdot{ }^{37,51}$

4. Elevated cAMP can increase IL-10 levels and inhibit the secretion of TNF-a and IL-12 by antigen-presenting cells, such as dendritic cells, to reduce inflammation.

\section{Pulmonary ventilation function}

$\mathrm{FEV}_{1}$ refers to the volume of maximum expiratory gas within 1 second after maximum inspiration. In patients with $\mathrm{ACO}$, $\mathrm{FEV}_{1} / \mathrm{FVC}<0.7$ and the pulmonary ventilation function are limited. Undoubtedly, improving the pulmonary ventilation will be beneficial for treating patients with ACO. ${ }^{12}$ Statistical and data analyses showed that roflumilast could increase the average $\mathrm{FEV}_{1}$ to $53.52 \mathrm{~mL}$ in patients with COPD. ${ }^{25}$ The studies of Bateman et al showed that roflumilast could improve ACO pulmonary ventilation and that $500 \mu \mathrm{g} /$ day gave more pronounced results than $100 \mu \mathrm{g} /$ day for $\mathrm{FEV}_{1}$ improvement. The specified asthma indicators were as follows: an increase in $\mathrm{FEV}_{1}>15 \%$ after bronchodilator use, or rheology $>20 \%$ of peak during testing at 1 week. ${ }^{52}$ After performing statistical analysis, Marsh et al proved that the probability of COPD combined with asthma was $55.2 \% .{ }^{53}$ Compared with patients with asthma and COPD, patients with ACO have worse basal lung function and decreased FEV $1 .{ }^{54}$ Current studies show that $\mathrm{FEV}_{1}$ can be used as a diagnostic indicator of ACO.

\section{Airway remodeling}

Airway remodeling in asthma is mainly manifested as airway mucinous epithelial metaplasia, fibrosis, subepithelial pachydermodactyly, airway smooth muscle cell proliferation, and vascular proliferation, while in COPD, it is mainly manifested as small airway goblet cell hyperplasia. Currently, ACO patients with airway remodeling typically exhibit changes in airway wall structure, and airway power stagnation or stiffness. Compared to patients with asthma and COPD, the airway wall of patients with ACO was significantly thickened, which aggravated airway obstruction.

Changes in airway wall structure, airway contraction, and airway fibrosis can result in airway remodeling. Airway inflammation, to a certain extent, will also aggravate airway remodeling. ACO patients with airway wall structure manifest changes mainly due to fibronectin and $\operatorname{IgG}$ and $\operatorname{IgM}$ deposition. Roflumilast can improve airway contraction by the following mechanisms: roflumilast can reduce the production of IL-5 and the level of eosinophil induced by allergen in sputum, affect the survival of B cells and the activation of eosinophils, so that the production of cysteine leukotrienes, eosinophil peroxidase, and $\lg \mathrm{E}$ is decreased, thereby inhibiting mast cell proliferation, reducing the changes in airway structure, and decreasing the bronchoconstriction induced by allergens. ${ }^{38}$ Studies have shown that roflumilast can significantly reduce the level of pulmonary fibrosis in patients with ACO, which may be related to its ability to significantly inhibit the proliferation and metastasis of fibroblasts induced by stem cell factor. Currently, it has been confirmed that roflumilast can cope with the tumor growth factor- $\beta$-induced fibroblast activation. ${ }^{49}$ Additionally, roflumilast can relieve the airway inflammation of $\mathrm{ACO}$, which is equivalent to indirectly relieving the airway remodeling of ACO. Roflumilast can increase the level of intracellular cAMP, thereby inhibiting the release of ROS from neutrophils and eosinophils. This pathway leads to decrease of myofibroblast levels and improves the severity of fibrosis through inhibiting epithelial to mesenchymal transition. Meanwhile, the increase in cAMP can activate cystic fibrosis transmembrane conductance regulator, thus enhancing the secretion of chloride in cross bronchial epithelial cells and the scavenging ability of mucus cilia.

\section{Improved mucociliary function}

Several experiments have demonstrated that roflumilast can increase the frequency of ciliary movement in the central and lateral bronchial trachea of murine lung slices in a dose-dependent manner. ${ }^{37}$ It has been found that roflumilast can maintain the number of bronchial cilia epithelial cells, ensure normal ciliary movement, increase the frequency of epithelial mucosal turnover, and improve mucus cilia function to alleviate mucus hypersecretion in patients with ACO by restraining the inflammatory cells from releasing IL-3 (by elevating cAMP level) and affecting the eosinophil cationic protein released by eosinophils. ${ }^{28}$ Additionally, roflumilast can inhibit the EPK, JNK pathway in the epithelial cells, reduce the expression of MUC5AC in the lung tissue, 
and enhance the cystic fibrosis transmembrane conductance regulator activity of its cells, thereby enhancing the mucus cleavage ability and alleviating mucus hypersecretion in patients with $\mathrm{ACO} .{ }^{37}$

\section{AHR}

Studies have shown that oral administration of $1,000 \mathrm{mg}$ daily can reduce the AHR of patients with asthma, and it also has a certain therapeutic effect on AHR in ACO ${ }^{27}$ The currently known mechanism by which roflumilast reduces AHR is that roflumilast can reduce the production of IL-5 and further affect the activation of B cells and eosinophils, ${ }^{28}$ thereby reducing the production of cysteine leukotrienes and eosinophil peroxidase, leading to the alleviation of AHR in patients with ACO. In addition, roflumilast could inhibit the immune response and relaxation of smooth muscle to improve AHR by increasing intracellular cAMP concentration.

In summary, roflumilast could act through multiple pathways of ACO pathogenesis with multiple links. The specific mechanism of roflumilast is shown in Figure 1. Also, the signaling pathway of cAMP in the treatment of ACO is shown in Figure 2. It could not only act on some cytokines directly, but also reduce airway inflammation, airway remodeling, and other ACO symptoms by acting on neutrophils, eosinophils, and other cells. Roflumilast has a pharmacological foundation for the treatment of ACO. It is considered a novel and effective method for treating ACO in both in vivo and in vitro experiments. In the event that there are no effective agents to control ACO symptoms, the use of roflumilast for clinical treatment of ACO has attracted considerable attention.

\section{Conclusion}

The role of roflumilast in asthma and COPD has been confirmed. As for the curative effect of ACO, an animal experiment conducted by Murad et $\mathrm{al}^{47}$ showed that roflumilast could significantly reduce the airway inflammation and symptoms such as tracheal contraction in ACO. Roflumilast has already entered Phase III clinical trials and is a promising agent for the clinical treatment of ACO. Knowledge on roflumilast's mechanism is gradually increasing, and mastery of this mechanism could undoubtedly help us to understand more of this agent's biological functions. However, the specific curative effects of roflumilast in $\mathrm{ACO}$, whether

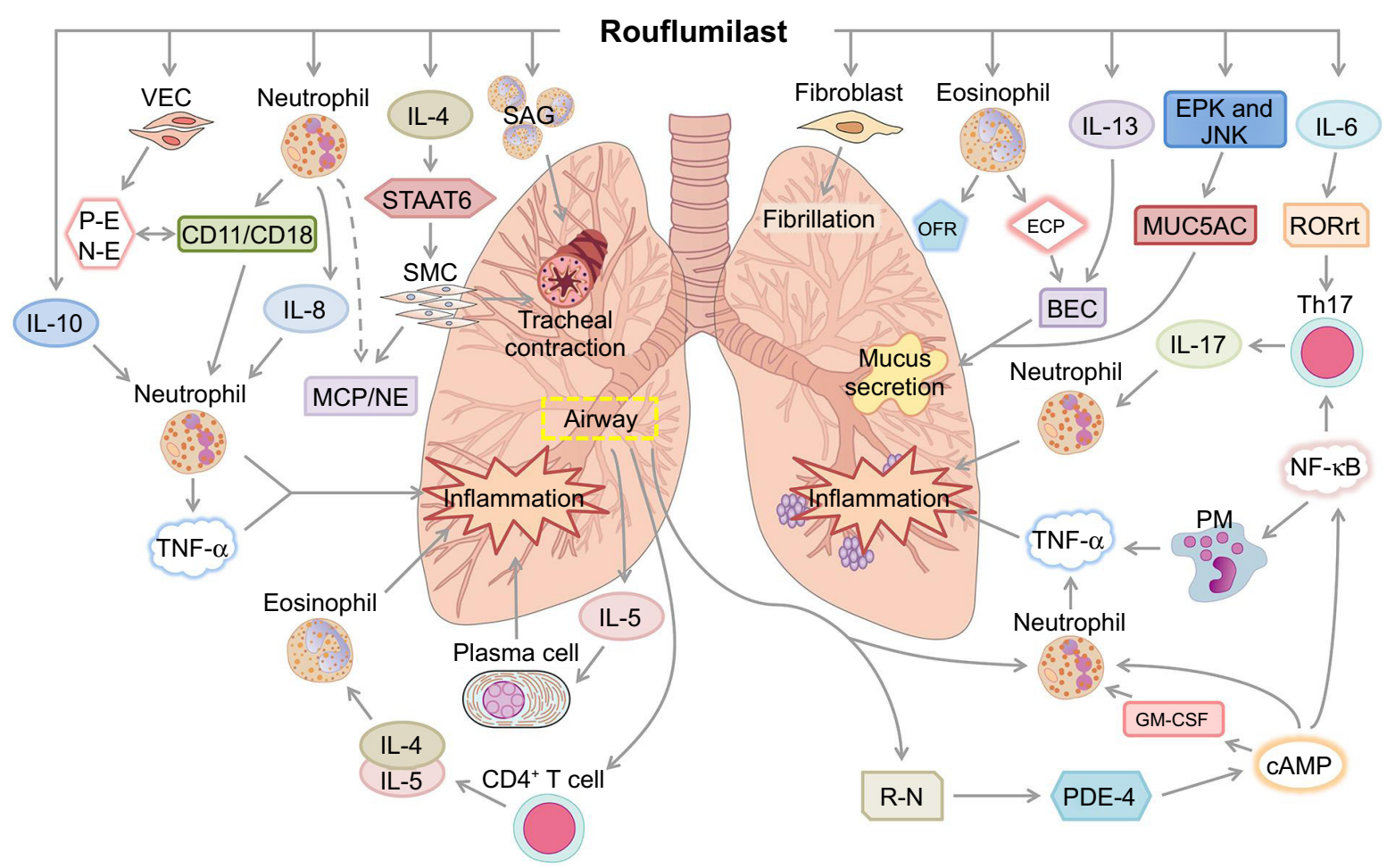

Figure I Pharmacological mechanism of roflumilast in ACO.

Abbreviations: ACO, asthma-COPD overlap; BEC, bronchial epithelial cells; cAMP, cyclic adenosine monophosphate; GM-CSF, granulocyte-macrophage colony stimulating factor; IL-X, interleukin-X; MUC5AC, mucin 5AC, oligomeric mucus/gel-forming; N-E, N-selectin; NF- KB, nuclear factor-kappa B; OFR, oxygen free radicals; PDE-4, phosphodiesterase-4 inhibitor; P-E, P-selectin; PM, pulmonary macrophage; R-N, roflumilast N-oxide; RORrt, retinoid-related orphan receptor- $\gamma$; SAG, sputum acid granulocytes; SMC, smooth muscle cells; STAAT6, signal transducer and activator of transcription 6; ThI7, T helper 17 lymphocytes; TNF- $\alpha$, tumor necrosis factor-alpha; VEC, vascular endothelial cells. 


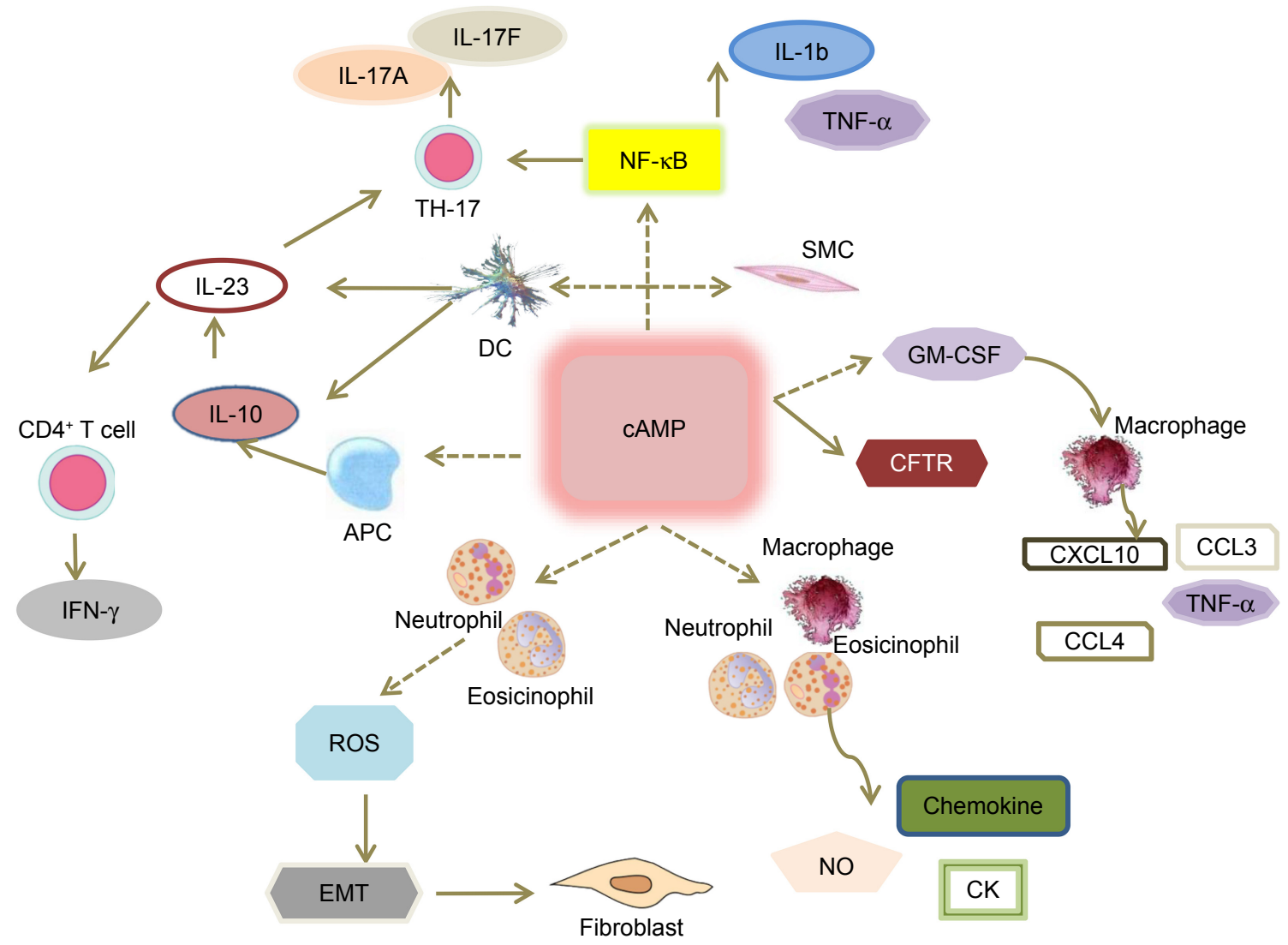

Figure 2 The signaling pathway of CAMP in the treatment of ACO.

Note: The dotted line represents suppression, and the solid line represents promotion.

Abbreviations: ACO, asthma-COPD overlap; APC, antigen-presenting cell; cAMP, cyclic adenosine monophosphate; CCL-X, chemokine C-C-motif ligand-X; CFTR, cystic fibrosis transmembrane conductance regulator; CK, cytokine; CXCLI0, C-X-C motif chemokine I0; DC, dendritic cell; EMT, epithelial to mesenchymal transition; GM-CSF, granulocyte-macrophage colony stimulating factor; IFN- $\gamma$, interferon- $\gamma$; IL-X, interleukin-X; NF- $\mathrm{B}$, nuclear factor-kappa B; NO, nitric oxide; ROS, reactive oxygen species; SMC, smooth muscle cells; ThI7, T-helper 17 lymphocytes; TNF- $\alpha$, tumor necrosis factor-alpha.

roflumilast can cause complications, the optimal dosage, and the appropriate targets and combinations remain unknown. Therefore, a multicenter, large-sample survey is needed for further verification.

\section{Disclosure}

The authors report no conflicts of interest in this work.

\section{References}

1. Mcnaughton A, Weatherall M, Williams M, et al. Sing Your Lungs Out-a community singing group for chronic obstructive pulmonary disease: a 1-year pilot study. BMJ Open. 2017;7(1):e014151.

2. Global Initiative for Asthma [homepage on the Internet]. Global Strategy for Asthma Management and Prevention; 2017. Available from: www. ginasthma.org

3. de Marco R, Pesce G, Marcon A, et al. The coexistence of asthma and chronic obstructive pulmonary disease (COPD): prevalence and risk factors in young, middle-aged and elderly people from the general population. PLoS One. 2013;8(5):e62985.

4. Kang J, Yao W, Cai B, Chen P, Ling X, Shang H. Current situation of asthma-COPD overlap syndrome (ACOS) in Chinese patients older than 40 years with airflow limitation: rationale and design for a multicenter, cross-sectional trial (study protocol. J Thorac Dis. 2016;8(12): 3744-3751.
5. Wurst KE, Kelly-Reif K, Bushnell GA, Pascoe S, Barnes N. Understanding asthma-chronic obstructive pulmonary disease overlap syndrome. Respir Med. 2016;110:1-11.

6. Turner AM, Tamasi L, Schleich F, et al. Clinically relevant subgroups in COPD and asthma. Eur Respir Rev. 2015;24(136):283-298.

7. Barrecheguren M, Esquinas C, Miravitlles M. The asthma-chronic obstructive pulmonary disease overlap syndrome (ACOS): opportunities and challenges. Curr Opin Pulm Med. 2015;21(1):74-79.

8. Gibson PG, Simpson JL. The overlap syndrome of asthma and COPD: what are its features and how important is it? Thorax. 2009;64(8):728-735.

9. Gauvreau GM, Boulet LP, Schmid-Wirlitsch C, et al. Roflumilast attenuates allergen-induced inflammation in mild asthmatic subjects. Respir Res. 2011;12:140.

10. Louie S, Zeki AA, Schivo M, et al. The asthma-chronic obstructive pulmonary disease overlap syndrome: pharmacotherapeutic considerations. Expert Rev Clin Pharmacol. 2013;6(2):197-219.

11. Hardin M, Silverman EK, Barr RG, et al; COPD Gene Investigators The clinical features of the overlap between COPD and asthma. Respir Res. 2011;12:127.

12. Padrão E, Araújo D, Todo Bom A, Bom AT, et al. Asthma-COPD overlap: a Portuguese survey. Pulmonology. 2018.

13. Lee HY, Kang JY, Yoon HK, et al. Clinical characteristics of asthma combined with COPD feature. Yonsei Med J. 2014;55(4):980-986.

14. Perez DLL, Cosío BG, Miravitlles M; Plaza VCHACOS Study Group. Accuracy of a new algorithm to identify asthma-COPD overlap (ACO) patients in a cohort of patients with chronic obstructive airway disease. Arch Bronconeumol. 2018;54(4):198-204. 
15. Mcdonald VM, Simpson JL, Higgins I, Gibson PG. Multidimensional assessment of older people with asthma and COPD: clinical management and health status. Age Ageing. 2011;40(1):42-49.

16. Barnes NC. The properties of inhaled corticosteroids: similarities and differences. Prim Care Respir J. 2007;16(3):149-154.

17. Hou G, Kang J. More attention to comprehensive assessment and individualized therapy of chronic obstructive pulmonary disease. $J$ Transl Int Med. 2015;3(2):39-42.

18. Mokry J, Urbanova A, Medvedova I, et al. Effects of tadalafil (PDE5 inhibitor) and roflumilast (PDE4 inhibitor) on airway reactivity and markers of inflammation in ovalbumin-induced airway hyperresponsiveness in guinea pigs. J Physiol Pharmacol. 2017;68(5): 721-730.

19. Meltzer EO, Chervinsky P, Busse W, et al. Roflumilast for asthma: Efficacy findings in placebo-controlled studies. Pulm Pharmacol Ther. 2015;35 Suppl(Suppl):S20-S27.

20. Barnette MS. Phosphodiesterase 4 (PDE4) inhibitors in asthma and chronic obstructive pulmonary disease (COPD). Prog Drug Res. 1999;53: 193-229.

21. Inagaki N, Miura T, Daikoku M, Nagai H, Koda A. Inhibitory effects of beta-adrenergic stimulants on increased vascular permeability caused by passive cutaneous anaphylaxis, allergic mediators, and mediator releasers in rats. Pharmacology. 1989;39(1):19-27.

22. Manning CD, Burman M, Christensen SB, et al. Suppression of human inflammatory cell function by subtype-selective PDE4 inhibitors correlates with inhibition of PDE4A and PDE4B. Br J Pharmacol. 1999; 128(7):1393-1398.

23. Ferreira J, Drummond M, Pires N, et al; GI DPOC-Grupo de Interesse na Doença Pulmonar Obstrutiva Crónica. Optimal treatment sequence in COPD: Can a consensus be found? Rev Port Pneumol. 2016;22(1):39-49.

24. Bardin P, Kanniess F, Gauvreau G, Bredenbröker D, Rabe KF. Roflumilast for asthma: Efficacy findings in mechanism of action studies. Pulm Pharmacol Ther. 2015;35 Suppl:S4-S10.

25. Bateman ED, Bousquet J, Aubier M, Bredenbröker D, O'Byrne PM. Roflumilast for asthma: efficacy findings in non-placebo-controlled comparator and dosing studies. Pulm Pharmacol Ther. 2015; 35 Suppl:S11-S19.

26. Rennard SI, Calverley PM, Goehring UM, Bredenbröker D, Martinez FJ. Reduction of exacerbations by the PDE4 inhibitor roflumilast - the importance of defining different subsets of patients with COPD. Respir Res. 2011;12:18.

27. Luo P, Li S, Chen Y, et al. Efficiency and safety of roflumilast combined with long-acting bronchodilators on moderate-to-severe stable chronic obstructive pulmonary disease patients: a meta-analysis. J Thorac Dis. 2016;8(9):2638-2645

28. Wedzicha JA, Calverley PMA, Albert RK, et al. Prevention of COPD exacerbations: a European Respiratory Society/American Thoracic Society guideline. Eur Respir J. 2017;50(3):1602265.

29. Hanania NA, Calverley PM, Dransfield MT, et al. Pooled subpopulation analyses of the effects of roflumilast on exacerbations and lung function in COPD. Respir Med. 2014;108(2):366-375.

30. Chong J, Poole P, Leung B, Black PN. Phosphodiesterase 4 inhibitors for chronic obstructive pulmonary disease. Cochrane Database Syst Rev. 2011;5:CD002309.

31. Kankaanranta H, Harju T, Kilpeläinen M, et al. Diagnosis and pharmacotherapy of stable chronic obstructive pulmonary disease: the finnish guidelines. Basic Clin Pharmacol Toxicol. 2015;116(4): 291-307.

32. Yan JH, Gu WJ, Pan L. Efficacy and safety of roflumilast in patients with stable chronic obstructive pulmonary disease: a meta-analysis. Pulm Pharmacol Ther. 2014;27(1):83-89.

33. Bredenbroker D, Syed J, Leichtl S, Rathgeb F, Wurst W. Roflumilast, a new orally active, selective phosphodiesterase 4 inhibitor, is effective in the treatment of chronic obstructive pulmonary disease. Eur Respir J. 2002;20(suppl 38):374S (abstr).
34. Rabe KF, Chapman KR, Jubert J, Vetter N, Witte S, Bredenbroeker D. Roflumilast, a novel selective phosphodiesterase 4 inhibitor improves lung function in patients with moderate to severe COPD. Am J Respir Crit Care Med. 2004;169:A518.

35. Lipworth BJ. Phosphodiesterase-4 inhibitors for asthma and chronic obstructive pulmonary disease. Lancet. 2005;365(9454):167-175.

36. Hatzelmann A, Morcillo EJ, Lungarella G, et al. The preclinical pharmacology of roflumilast - a selective, oral phosphodiesterase 4 inhibitor in development for chronic obstructive pulmonary disease. Pulm Pharmacol Ther. 2010;23(4):235-256.

37. Pan L, Guo YZ, Zhang B, Yan JH. Does roflumilast improve dyspnea in patients with chronic obstructive pulmonary disease? A meta-analysis. J Thorac Dis. 2013;5(4):422-429.

38. Louw C, Williams Z, Venter L, et al. Roflumilast, a phosphodiesterase 4 inhibitor, reduces airway hyperresponsiveness after allergen challenge. Respiration. 2007;74(4):411-417.

39. Herbert C, Hettiaratchi A, Webb DC, Thomas PS, Foster PS, Kumar RK. Suppression of cytokine expression by roflumilast and dexamethasone in a model of chronic asthma. Clin Exp Allergy. 2008;38(5):847-856.

40. Timmer $\mathrm{W}$, Leclerc V, Birraux G, et al. The new phosphodiesterase 4 inhibitor roflumilast is efficacious in exercise-induced asthmaand leads to suppression of LPS-stimulated TNF-alpha ex vivo. J Clin Pharmacol. 2002;42(3):297-303.

41. van Schalkwyk EM, van Heerden K, Bredenbroker D, et al. Dosedependent inhibitory effect of roflumilast, a new, orally active, selective phosphodiesterase 4 inhibitor, on allergen-induced early and late asthmatic reaction. Eur Respir J. 2002;20(suppl 38):110S.

42. Leichtl S, Schmid-Wirlitsch C, Bredenbröker D, Rathgeb F, Wurst W. Roflumilast, a new orally active, selective phosphodiesterase 4 inhibitor is effective in the treatment of asthma. Eur Respir J. 2002;20(suppl 38): $303 \mathrm{~S}$ (abstr).

43. Shantakumar S, Pwu RF, D'Silva L, et al. Burden of asthma and COPD overlap (ACO) in Taiwan: a nationwide population-based study. BMC Pulm Med. 2018;18(1):16.

44. D'Silva L, Hassan N, Wang HY, et al. Heterogeneity of bronchitis in airway diseases in tertiary care clinical practice. Can Respir J. 2011; 18(3):144-148.

45. Papaiwannou A, Zarogoulidis P, Porpodis K, et al. Asthma-chronic obstructive pulmonary disease overlap syndrome (ACOS): current literature review. J Thorac Dis. 2014;6 Suppl 1:S146-S151.

46. Mendes ES, Rebolledo P, Cadet L, Arana J, Scmid A, Wanner A. Effect of roflumilast on airway blood flow in COPD: a pilot study. Chronic Obstr Pulm Dis. 2017;4(4):262-264.

47. Murad HA, Habib HS, Rafeeq MM, Sulaiman MI, Abdulrahman AS, Khabaz MN. Co-inhalation of roflumilast, rather than formoterol, with fluticasone more effectively improves asthma in asthmatic mice. Exp Biol Med. 2017;242(5):516-526.

48. Grootendorst DC, Gauw SA, Verhoosel RM, et al. Reduction in sputum neutrophil and eosinophil numbers by the PDE4 inhibitor roflumilast in patients with COPD. Thorax. 2007;62(12):1081-1087.

49. Yalcin AD, Celik B, Yalcin AN. Omalizumab (anti-IgE) therapy in the asthma-COPD overlap syndrome (ACOS) and its effects on circulating cytokine levels. Immunopharmacol Immunotoxicol. 2016; 38(3):253-256.

50. Araújo D, Padrão E, Morais-Almeida M, et al. Asthma-chronic obstructive pulmonary disease overlap syndrome - literature review and contributions towards a Portuguese consensus. Rev Port Pneumol. 2017; 23(2):90-99.

51. Kawamatawong T. Roles of roflumilast, a selective phosphodiesterase 4 inhibitor, in airway diseases. J Thorac Dis. 2017;9(4): 1144-1154.

52. Bateman E, Singh D, Smith D, et al. Efficacy and safety of tiotropium Respimat SMI in COPD in two 1-year randomized studies. Int J Chron Obstruct Pulmon Dis. 2010;5:197-208.

53. Marsh SE, Travers J, Weatherall M, et al. Proportional classifications of COPD phenotypes. Thorax. 2008;63(9):761-767. 
54. Kim SW, Kim JH, Park CK, et al. Effect of roflumilast on airway remodelling in a murine model of chronic asthma. Clin Exp Allergy. 2016;46(5):754-763.

55. Barnes NC, Saetta M, Rabe KF. Implementing lessons learned from previous bronchial biopsy trials in a new randomized controlled COPD biopsy trial with roflumilast. BMC Pulm Med. 2014;14:9.
56. Jianli $\mathrm{P}$, Lun $\mathrm{H}$, Ling $\mathrm{O}$, et al. [Clinical values of interleukin-17 in the differentiation of asthma, chronic obstructive pulmonary disease and asthma chronic obstructive pulmonary disease overlap syndrome].Chin J Lung Dis(Electronic Edition). 2016;5(9):479-483. Chinese.

\section{Publish your work in this journal}

Drug Design, Development and Therapy is an international, peerreviewed open-access journal that spans the spectrum of drug design and development through to clinical applications. Clinical outcomes, patient safety, and programs for the development and effective, safe, and sustained use of medicines are the features of the journal, which has also been accepted for indexing on PubMed Central. The manuscript management system is completely online and includes a very quick and fair peer-review system, which is all easy to use. Visit http://www.dovepress.com/testimonials.php to read real quotes from published authors.

Submit your manuscript here: http://www.dovepress.com/drug-design-development-and-therapy-journal 